\title{
Double Hodge Theory for a Particle on Torus
}

\author{
Vipul Kumar Pandey and Bhabani Prasad Mandal \\ Department of Physics, Banaras Hindu University, Varanasi 221005, India \\ Correspondence should be addressed to Vipul Kumar Pandey; vipulvaranasi@gmail.com
}

Received 1 July 2017; Accepted 31 October 2017; Published 27 November 2017

Academic Editor: Edward Sarkisyan-Grinbaum

Copyright (c) 2017 Vipul Kumar Pandey and Bhabani Prasad Mandal. This is an open access article distributed under the Creative Commons Attribution License, which permits unrestricted use, distribution, and reproduction in any medium, provided the original work is properly cited. The publication of this article was funded by SCOAP ${ }^{3}$.

\begin{abstract}
We investigate all possible nilpotent symmetries for a particle on torus. We explicitly construct four independent nilpotent BRST symmetries for such systems and derive the algebra between the generators of such symmetries. We show that such a system has rich mathematical properties and behaves as double Hodge theory. We further construct the finite field dependent BRST transformation for such systems by integrating the infinitesimal BRST transformation systematically. Such a finite transformation is useful in realizing the various theories with toric geometry.
\end{abstract}

\section{Introduction}

The formulation based on BRST symmetry [1-4] plays a crucial role in the discussion of quantization, renormalization, and unitarity and other aspects of gauge theories. The nilpotency nature of BRST transformation is mainly responsible for simplified treatment in all these discussions. Thus it is extremely important to find more and more nilpotent symmetry associated with any system to study, particularly the systems with constraints. Toric geometry which is generalization of the projective identification that defines $C P^{n}$ corresponding to the most general linear sigma model provides a scheme for constructing Calabi-Yau manifolds and their mirrors [5]. Recently, on the basis of boundary string field theory [6], the brane-antibrane system was exploited [7] in the toroidal background to investigate its thermodynamic properties associated with the Hagedorn temperature $[8,9]$. The Nahm transform and moduli spaces of $C P^{n}$ models were also studied on the toric geometry [10]. In a four-dimensional, toroidally compactified heterotic string, the electrically charged BPS-saturated states were shown to become massless along the hyper surfaces of enhanced gauge symmetry of a two-torus moduli subspace [11].

In the present work we investigate various possible nilpotent symmetries for a particle on torus. Usual BRST symmetry for a particle on torus has already been constructed [12]. In this work we construct four different nilpotent symmetries associated with this system, namely, BRST symmetry, antiBRST symmetry, dual BRST (also known as co-BRST) symmetry, and anti-dual BRST (also known as anti-co-BRST) symmetry [13-15]. We further construct two different bosonic symmetries using these nilpotent BRST symmetries and some discrete symmetries associated with ghost number are also written for such systems. Complete algebra satisfied by charges, which generate these symmetries, is derived. Deep mathematical connections of such system with Hodge theory [16-19] are established in this work. We found that the system of particle on a torus is realized as Hodge theory with respect to two different sets of operators. The generators for BRST, dual BRST symmetries, and generator for corresponding bosonic symmetries constructed out of BRST and dual BRST symmetries are analogous to exterior derivative, coexterior derivative, and Laplace operator in Hodge theory [20-28]. On the other hand the charges corresponding to anti-BRST symmetry, anti-dual BRST symmetry, and bosonic symmetry constructed out of these two BRST symmetries are also from set of de Rham cohomological operators. This indicates that the mathematical foundation of the theory of a particle on a torus is extremely rich.

We further extend the BRST transformation for this system by considering the BRST parameter as finite and field dependent. More than two decades ago Joglekar and Mandal introduced for the first time the concept of finite field dependent BRST (FFBRST) transformation [29], which 
had similar structure and properties of usual BRST transformation. However the path integral measure is not invariant due to finite nature of such transformation. It has been shown that by constructing suitable finite parameter one can calculate desirable Jacobian factor which under certain condition is added to the effective action of the theory. Thus FFBRST is capable of connecting generating functionals of two different effective theories. Because of these remarkable properties, FFBRST has become a useful tool of studying various field theoretic systems with BRST symmetry and it has found many applications [30-43]. We have constructed FFBRST transformation for the system of particle on torus to show the connection between two theories on torus with different gauge fixing. Now we present the plan of this manuscript.

We start with the brief introduction about the free particle on the surface of torus in Section 2. Hamiltonian formulation for this theory is presented in Section 3. In Section 4 the BFV formulation for this model has been discussed and BRST symmetry for such model has been constructed. In Section 5 the other nilpotent symmetry transformations for same system have been constructed. Co-BRST and anti-coBRST have been discussed in Section 6. Other symmetries have been discussed in Section 7. The connection between algebra satisfied by the nilpotent charges and de Rham cohomological operators of differential geometry is shown in Section 8. In Section 9 we introduce FFBRST transformation and in the next section we connect theory in different gauges using FFBRST transformations. We conclude our results in Section 10.

\section{Free Particle on Surface of Torus}

BRST Symmetry for free particle system on toric geometry has already been studied using BFV formalism in [12]. Here we review some parts of the work in [12] relevant to our calculation in later chapters. A particle moving freely on the surface of a torus is described by Lagrangian [12]:

$$
L_{0}=\frac{1}{2} m \dot{r}^{2}+\frac{1}{2} m r^{2} \dot{\theta}^{2}+\frac{1}{2} m(b+r \sin \theta)^{2} \dot{\phi}^{2},
$$

where $(r, \theta, \phi)$ are toroidal coordinates related to Cartesian coordinates as

$$
\begin{aligned}
& x=(b+r \sin \theta) \cos \phi, \\
& y=(b+r \sin \theta) \sin \phi, \\
& z=r \cos \theta .
\end{aligned}
$$

Here we have considered a torus with axial circle in the $x-y$ plane centered at the origin, of radius $b$, having a circular cross section of radius $r$. The angle $\theta$ ranges from 0 to $2 \pi$ and the angle $\phi$ from 0 to $2 \pi$. Since the particle moves on the surface of torus of radius $r$, it is constrained to satisfy

$$
\Omega_{1}=r-a \approx 0 \text {. }
$$

The canonical Hamiltonian corresponding to the Lagrangian in (1) with the above constraint is then written as

$$
H_{0}=\frac{p_{r}^{2}}{2 m}+\frac{p_{\theta}^{2}}{2 m r^{2}}+\frac{p_{\phi}^{2}}{2 m(b+r \sin \theta)^{2}}+\lambda(r-a),
$$

where $p_{r}, p_{\theta}$, and $p_{\phi}$ are the canonical momenta conjugate to the coordinate $r, \theta$, and $\phi$, respectively, given by

$$
\begin{aligned}
& p_{r}=m \dot{r}, \\
& p_{\theta}=m r^{2} \dot{\theta}, \\
& p_{\phi}=m(b+r \sin \theta)^{2} \dot{\phi} .
\end{aligned}
$$

The time evolution of the constraint $\Omega_{1}$ yields the secondary constraint as

$$
\Omega_{2}=p_{r} \approx 0
$$

\section{Wess-Zumino Term and Hamiltonian Formulation}

To construct a gauge invariant theory corresponding to the gauge noninvariant model in (4), we introduce the WessZumino term [28] in the Lagrangian density $\mathscr{L}$. For this purpose we enlarge the Hilbert space of the theory by introducing a new quantum field $\eta$, called Wess-Zumino field, through the redefinition of fields $r$ and $\lambda$ in the original Lagrangian density $\mathscr{L}$ as follows:

$$
\begin{aligned}
& r \longrightarrow r-\eta ; \\
& \lambda \longrightarrow \lambda+\dot{\eta} .
\end{aligned}
$$

With this redefinition of the fields, the modified Lagrangian density becomes

$$
\begin{aligned}
\mathscr{L}^{\mathscr{g}}= & \frac{1}{2} m(\dot{r}-\dot{\eta})^{2}+\frac{1}{2} m(r-\eta)^{2} \dot{\theta}^{2} \\
& +\frac{1}{2} m(b+(r-\eta) \sin \theta)^{2} \dot{\phi}^{2} \\
& -(\lambda+\dot{\eta})(r-a-\eta) .
\end{aligned}
$$

Canonical momenta corresponding to this modified Lagrangian density are then given by

$$
\begin{aligned}
& p_{r}=m(\dot{r}-\dot{\eta}), \\
& p_{\eta}=-(m(\dot{r}-\dot{\eta})+(r-a-\eta)), \\
& p_{\lambda}=0 \\
& p_{\theta}=m(r-\eta)^{2} \dot{\theta}, \\
& p_{\phi}=m(b+(r-\eta) \sin \theta)^{2} \dot{\phi} .
\end{aligned}
$$

The primary constraint for this extended theory is

$$
\psi_{1} \equiv p_{\lambda} \approx 0
$$


The Hamiltonian density corresponding to $\mathscr{L}^{\mathscr{F}}$ is written as

$$
H^{I}=p_{r} \dot{r}+p_{\eta} \dot{\eta}+p_{\theta} \dot{\theta}+p_{\phi} \dot{\phi}+p_{\lambda} \dot{\lambda}-\mathscr{L}^{\mathscr{F}} \text {. }
$$

The total Hamiltonian density after the introduction of a Lagrange multiplier field $u$ corresponding to the primary constraint $\psi_{1}$ is then obtained as

$$
\begin{aligned}
H_{T}^{I}= & \frac{p_{r}^{2}}{2 m}+\frac{p_{\theta}^{2}}{2 m(r-\eta)^{2}}+\frac{p_{\phi}^{2}}{2 m(b+(r-\eta) \sin \theta)^{2}} \\
& +\lambda\left(p_{r}+p_{\eta}\right)+u p_{\lambda} .
\end{aligned}
$$

Following Dirac's method of constraint analysis [44-47], we obtain secondary constraint

$$
\psi_{2} \equiv\left(p_{\eta}+p_{r}\right) \approx 0
$$

In next two sections, we extend this constrained theory to study the nilpotent symmetries associated with this theory.

\section{BFV Formulation for Free Particle on the Surface of Torus}

To discuss all possible nilpotent symmetries we further extend the theory using BFV formalism [48-53]. In the $\mathrm{BFV}$ formulation associated with this system, we introduce a pair of canonically conjugate ghost fields $(c, p)$ with ghost numbers 1 and -1 , respectively, for the primary constraint $p_{\lambda} \approx 0$ and another pair of ghost fields $(\bar{c}, \bar{p})$ with ghost numbers -1 and 1 , respectively, for the secondary constraint, $\left(p_{\eta}+p_{r}\right) \approx 0$. The effective action for a particle on surface of the torus in extended phase space is then written as

$$
\begin{aligned}
S_{\mathrm{eff}} & =\int d^{4} x\left[p_{r} \dot{r}+p_{\eta} \dot{\eta}+p_{\theta} \dot{\theta}+p_{\phi} \dot{\phi}-p_{\lambda} \dot{\lambda}-\frac{p_{r}^{2}}{2 m}\right. \\
& -\frac{p_{\theta}^{2}}{2 m(r-\eta)^{2}}-\frac{p_{\phi}^{2}}{2 m(b+(r-\eta) \sin \theta)^{2}}+\dot{c} p+\dot{\bar{c}} \bar{p} \\
& \left.-\left\{Q_{b}, \psi\right\}\right],
\end{aligned}
$$

where $Q_{b}$ is the BRST charge and $\psi$ is the gauge fixed fermion. This effective action is invariant under BRST transformation generated by $Q_{b}$ which is constructed by using constraints in the theory as

$$
Q_{b}=i c\left(p_{r}+p_{\eta}\right)-i \bar{p} p_{\lambda}
$$

The canonical brackets for all dynamical variables are written as

$$
\begin{aligned}
{\left[r, p_{r}\right] } & =\left[\theta, p_{\theta}\right]=\left[\phi, p_{\phi}\right]=\left[\eta, p_{\eta}\right]=\left[\lambda, p_{\lambda}\right]=\{\bar{c}, \dot{c}\} \\
& =i \\
\{c, \dot{\bar{c}}\} & =-i,
\end{aligned}
$$

where rest of the brackets are zero. Now, the nilpotent BRST transformation, using the relation $s_{b} \phi=\left[\phi, Q_{b}\right]_{ \pm}( \pm$sign represents the fermionic and bosonic nature of the fields $\phi$ ), is explicitly written as

$$
\begin{aligned}
s_{b} r & =-c, \\
s_{b} \lambda & =\bar{p}, \\
s_{b} \bar{p} & =0, \\
s_{b} \theta & =-c \\
s_{b} p_{\phi} & =0, \\
s_{b} p_{\theta} & =0, \\
s_{b} p & =\left(p_{r}+p_{\eta}\right) \\
s_{b} \bar{c} & =p_{\lambda}, \\
s_{b} p_{\lambda} & =0, \\
s_{b} c & =0 .
\end{aligned}
$$

In BFV formulation the generating functional is independent of gauge fixed fermion [48-53]; hence we have liberty to choose it in the convenient form as

$$
\psi=p \lambda+\bar{c}\left(r+\eta+\frac{p_{\lambda}}{2}\right)
$$

Putting the value of $\psi$ in (14) and using (15) and (16), we obtain

$$
\begin{aligned}
S_{\mathrm{eff}} & =\int d^{4} x\left[p_{r} \dot{r}+p_{\eta} \dot{\eta}+p_{\theta} \dot{\theta}+p_{\phi} \dot{\phi}-p_{\lambda} \dot{\lambda}-\frac{p_{r}^{2}}{2 m}\right. \\
& -\frac{p_{\theta}^{2}}{2 m(r-\eta)^{2}}-\frac{p_{\phi}^{2}}{2 m(b+(r-\eta) \sin \theta)^{2}}+\dot{c} p+\dot{\bar{c}} \bar{p} \\
& \left.+\lambda\left(p_{r}+p_{\eta}\right)+2 c \bar{c}-\bar{p} p+p_{\lambda}\left(r+\eta+\frac{p_{\lambda}}{2}\right)\right]
\end{aligned}
$$

and the generating functional for this effective theory is represented as

$$
Z_{\psi}=\int D \phi \exp \left[i S_{\mathrm{eff}}\right]
$$

Now integrating this generating functional over $p$ and $\bar{p}$, we get

$$
\begin{aligned}
Z_{\psi} & =\int D \phi^{\prime} \exp \left[i \int d ^ { 4 } x \left[p_{r} \dot{r}+p_{\eta} \dot{\eta}+p_{\theta} \dot{\theta}+p_{\phi} \dot{\phi}\right.\right. \\
& -p_{\lambda} \dot{\lambda}-\frac{p_{r}^{2}}{2 m}-\frac{p_{\theta}^{2}}{2 m(r-\eta)^{2}}
\end{aligned}
$$




$$
\begin{aligned}
& -\frac{p_{\phi}^{2}}{2 m(b+(r-\eta) \sin \theta)^{2}}+\dot{\bar{c}} \dot{c}+\lambda\left(p_{r}+p_{\eta}\right)+2 c \bar{c} \\
& \left.\left.+p_{\lambda}\left(r+\eta+\frac{p_{\lambda}}{2}\right)\right]\right]
\end{aligned}
$$

where $D \phi^{\prime}$ is the path integral measure for effective theory when integrations over fields $p$ and $\bar{p}$ are carried out. Further integrating over field $p_{\lambda}$ we obtain an effective generating functional as

$$
\begin{aligned}
& Z_{\psi} \\
& =\int D \phi^{\prime \prime} \exp \left[i \int d^{4} x\left[p_{r} \dot{r}+p_{\eta} \dot{\eta}+p_{\theta} \dot{\theta}+p_{\phi} \dot{\phi}-\frac{p_{r}^{2}}{2 m}-\frac{p_{\theta}^{2}}{2 m(r-\eta)^{2}}-\frac{p_{\phi}^{2}}{2 m(b+(r-\eta) \sin \theta)^{2}}+\dot{\bar{c}} \dot{c}+\lambda\left(p_{r}+p_{\eta}\right)-2 \bar{c} c-\frac{(\dot{\lambda}-r-\eta)^{2}}{2}\right]\right]
\end{aligned}
$$

where $D \phi^{\prime \prime}$ is the path integral measure corresponding to all the dynamical variables involved in the effective action. The BRST symmetry transformation for this effective theory is written as

$$
\begin{aligned}
s_{b} r & =-c, \\
s_{b} \lambda & =\dot{c}, \\
s_{b} \eta & =-c \\
s_{b} p_{r} & =0, \\
s_{b} p_{\eta} & =0 \\
s_{b} \bar{c} & =-(\dot{\lambda}-\eta-r), \\
s_{b} c & =0 .
\end{aligned}
$$

These transformations are on shell nilpotent.

\section{Nilpotent Symmetries}

In this section we will study various other nilpotent symmetries of this model with particle on a torus [54]. For this purpose it is convenient to work using Nakanishi-Lautrup type auxiliary field $B$ which linearizes the gauge fixing part of the effective action in (22). The first-order effective action is then given by

$$
\begin{aligned}
S_{\text {eff }} & =\int d^{4} x\left[p_{r} \dot{r}+p_{\eta} \dot{\eta}+p_{\theta} \dot{\theta}+p_{\phi} \dot{\phi}-\frac{p_{r}^{2}}{2 m}\right. \\
& -\frac{p_{\theta}^{2}}{2 m(r-\eta)^{2}}-\frac{p_{\phi}^{2}}{2 m(b+(r-\eta) \sin \theta)^{2}}+\dot{\bar{c}} \dot{c} \\
& \left.+\lambda\left(p_{r}+p_{\eta}\right)-2 \bar{c} c-B(\dot{\lambda}-r-\eta)+\frac{B^{2}}{2}\right] .
\end{aligned}
$$

We can easily show that this action is invariant under the following off-shell nilpotent BRST transformation:

$$
\begin{aligned}
& s_{b} r=-c \\
& s_{b} \lambda=\dot{c}
\end{aligned}
$$

$$
\begin{aligned}
s_{b} \eta & =-c \\
s_{b} p_{r} & =0, \\
s_{b} p_{\eta} & =0, \\
s_{b} \theta & =0 \\
s_{b} \bar{c} & =B, \\
s_{b} \bar{c} & =0, \\
s_{b} p_{\phi} & =0 \\
s_{b} \phi & =0, \\
s_{b} p_{\theta} & =0 .
\end{aligned}
$$

Corresponding anti-BRST transformation for this theory is then written by interchanging the role of ghost and anti-ghost field as

$$
\begin{gathered}
s_{a b} r=-\bar{c}, \\
s_{a b} \lambda=\dot{\bar{c},} \\
s_{a b} \eta=-\bar{c} \\
s_{a b} p_{r}=0, \\
s_{a b} p_{\eta}=0, \\
s_{a b} p_{\phi}=0 \\
s_{a b} c=-B, \\
s_{a b} \bar{c}=0, \\
s_{a b} \theta=0 \\
s_{a b} \phi=0, \\
s_{a b} p_{\theta}=0 .
\end{gathered}
$$

The conserved BRST and anti-BRST charges $Q_{b}$ and $Q_{a b}$ which generate above BRST and anti-BRST transformations are written for this effective theory as

$$
Q_{b}=i c\left(p_{r}+p_{\eta}\right)-i p_{\lambda} \dot{c}
$$




$$
Q_{a b}=i \bar{c}\left(p_{r}+p_{\eta}\right)-i p_{\lambda} \dot{\bar{c}}
$$

Further by using the following equation of motion

$$
\begin{aligned}
B+\dot{p}_{r} & =0, \\
B+\dot{p}_{\eta} & =0 \\
\dot{r}-p_{r}+\lambda & =0 \\
\dot{B} & =p_{r}+p_{\eta}, \\
\dot{\bar{c}}+2 \bar{c} & =0, \\
\dot{c}+2 c & =0, \\
B+\dot{\lambda}-r-\eta & =0
\end{aligned}
$$

it is shown that these charges are constants of motion, that is, $\dot{Q}_{b}=0, \dot{Q}_{a b}=0$, and satisfy following relations:

$$
Q_{b} Q_{a b}+Q_{a b} Q_{b}=0
$$

To arrive to these relations, the canonical brackets (16) of the fields and the definition of canonical momenta have been used

$$
\begin{aligned}
& p_{\lambda}=B, \\
& p_{\bar{c}}=\dot{c}, \\
& p_{c}=-\dot{\bar{c}} .
\end{aligned}
$$

The physical states of theory are annihilated by the BRST and anti-BRST charges, leading to

$$
\begin{aligned}
\left.\left(p_{r}+p_{\eta}\right) \mid \text { phys }\right\rangle & =0 \\
\left.p_{\lambda} \mid \text { phys }\right\rangle & =0 .
\end{aligned}
$$

This implies that the operator form of the first class constraint $p_{\lambda} \approx 0$ and $\left(p_{r}+p_{\eta}\right) \approx 0$ annihilates the physical state of the theory. Thus the physicality criteria are consistent with Dirac's method of quantization.

\section{Co-BRST and Anti Co-BRST Symmetries}

In this section, we investigate two other nilpotent transformations, namely, co-BRST and anti-co-BRST transformation, which are also the symmetry of the effective action in (24). Further these transformations leave the gauge fixing term of the action invariant independently and the kinetic energy term (which remains invariant under BRST and anti-BRST transformations) transforms under it to compensate for the transformation of the ghost terms. These transformations are also called dual and anti-dual BRST transformation [13-15].

The nilpotent co-BRST transformation $\left(s_{d}^{2}=0\right)$ and antico-BRST transformation $\left(s_{a d}^{2}=0\right)$, which leave the effective action [in (24)] for a particle on torus invariant, are given by

$$
\begin{aligned}
s_{d} r & =-\frac{1}{2} \dot{\bar{c}}, \\
s_{d} \lambda & =-\bar{c}, \\
s_{d} \eta & =-\frac{1}{2} \dot{\bar{c}} \\
s_{d} p_{r} & =0, \\
s_{d} p_{\eta} & =0, \\
s_{d} \bar{c} & =0 \\
s_{d} c & =\frac{1}{2}\left(p_{r}+p_{\eta}\right), \\
s_{d} B & =0, \\
s_{a d} r & =-\frac{1}{2} \dot{c}, \\
s_{a d} \lambda & =-c, \\
s_{a d} \eta & =-\frac{1}{2} \dot{c} \\
s_{a d} p_{r} & =0, \\
s_{a d} B & =0 . \\
s_{a d} p_{\eta} & =0, \\
c & =0 \\
s_{a} & \\
s_{a} &
\end{aligned}
$$

These transformations are absolutely anticommuting as $\left\{S_{d}, S_{a d}\right\}=0$. The conserved charges for above symmetries are found using Noether's theorem and are written as

$$
\begin{aligned}
Q_{d} & =i \frac{1}{2}\left(p_{r}+p_{\eta}\right) \dot{\bar{c}}+i p_{\lambda} \bar{c} \\
Q_{a d} & =i \frac{1}{2}\left(p_{r}+p_{\eta}\right) \dot{c}+i p_{\lambda} c
\end{aligned}
$$

which generate the symmetry transformations in (32) and (33), respectively. It is easy to verify the following relations:

$$
\begin{gathered}
s_{d} Q_{d}=-\left\{Q_{d}, Q_{d}\right\}=0 \\
s_{a d} Q_{a d}=-\left\{Q_{a d}, Q_{a d}\right\}=0 \\
s_{d} Q_{a d}=-\left\{Q_{a d}, Q_{d}\right\}=0 \\
s_{a d} Q_{d}=-\left\{Q_{d}, Q_{a d}\right\}=0
\end{gathered}
$$

which reflect the nilpotency and anti-commutativity property of $s_{d}$ and $s_{a d}$ (i.e., $s_{d}^{2}=0, s_{a d}^{2}=0$ and $s_{d} s_{a d}+s_{a d} s_{d}=0$ ). 


\section{Other Symmetries}

In this section, we construct other symmetries related to this system. Two different bosonic symmetries are constructed out of four nilpotent symmetries. Discrete symmetry related to ghost number is also constructed.

7.1. Bosonic Symmetry. In this part we construct the bosonic symmetry out of these nilpotent BRST symmetries of the theory using [50-52]. The BRST $\left(s_{b}\right)$, anti-BRST $\left(s_{a b}\right)$, co$\operatorname{BRST}\left(s_{d}\right)$, and anti-co-BRST $\left(s_{a d}\right)$ symmetry operators satisfy the following algebra:

$$
\begin{aligned}
& \left\{s_{d}, s_{a d}\right\}=0, \\
& \left\{s_{b}, s_{a b}\right\}=0 \\
& \left\{s_{b}, s_{a d}\right\}=0, \\
& \left\{s_{d}, s_{a b}\right\}=0
\end{aligned}
$$

and we define bosonic symmetries $s_{w}$ and $s_{\bar{w}}$ as

$$
\begin{aligned}
& s_{w} \equiv\left\{s_{b}, s_{d}\right\}, \\
& s_{\bar{w}} \equiv\left\{s_{a b}, s_{a d}\right\} .
\end{aligned}
$$

The fields variables transform under bosonic symmetry $s_{w}$ as

$$
\begin{aligned}
s_{w} r & =-\frac{1}{2}\left(\dot{B}+p_{r}+p_{\eta}\right), \\
s_{w} \lambda & =-\frac{1}{2}\left(2 B-\dot{p}_{r}-\dot{p}_{\eta}\right) \\
s_{w} \eta & =-\frac{1}{2}\left(\dot{B}+p_{r}+p_{\eta}\right), \\
s_{w} p_{r} & =0 \\
s_{w} p_{\eta} & =0 \\
s_{w} c & =0 \\
s_{w} B & =0 \\
s_{w} \bar{c} & =0 .
\end{aligned}
$$

On the other hand transformation generated by $s_{\bar{w}}$ is

$$
\begin{aligned}
s_{\bar{w}} r & =-\frac{1}{2}\left(\dot{B}+p_{r}+p_{\eta}\right), \\
s_{\bar{w}} \lambda & =\frac{1}{2}\left(2 B-\dot{p}_{r}-\dot{p}_{\eta}\right) \\
s_{\bar{w}} \eta & =-\frac{1}{2}\left(\dot{B}+p_{r}+p_{\eta}\right), \\
s_{\bar{w}} p_{r} & =0, \\
s_{\bar{w}} p_{\eta} & =0 \\
s_{\bar{w}} c & =0, \\
s_{\bar{w}} B & =0 \\
s_{\bar{w}} \bar{c} & =0 .
\end{aligned}
$$

However the transformation generated by $s_{w}$ and $s_{\bar{w}}$ is not independent as it is easy to see from (38) and (39) that the operators $s_{w}$ and $s_{\bar{w}}$ satisfy the relation $s_{w}+s_{\bar{w}}=0$. This implies from (37) that

$$
\left\{s_{b}, s_{d}\right\}=s_{w}=-\left\{s_{a b}, s_{a d}\right\} .
$$

It is clear from above algebra that the operator $s_{w}$ analogous of the Laplacian operator in the language of differential geometry and the conserved charge for the above symmetry transformation is calculated as

$$
Q_{w}=-i\left[B^{2}+\frac{1}{2}\left(p_{r}+p_{\eta}\right)^{2}\right]
$$

which generates the transformation in (38).

Using equation of motion, it can readily be checked that

$$
\frac{d Q_{w}}{d t}=-i \int d x\left[2 B \dot{B}+\left(p_{r}+p_{\eta}\right)\left(\dot{p}_{r}+\dot{p}_{\eta}\right)\right]=0
$$

Hence $Q_{w}$ is the constant of motion for this theory.

7.2. Ghost Symmetry and Discrete Symmetry. Now we consider yet another kind of symmetry of this system called ghost symmetry discussed in [50]. The ghost numbers of the ghost and anti-ghost fields are 1 and -1 , respectively. Rest of the variables in the action of this theory have ghost number zero. Keeping this fact in mind we can introduce a scale transformation of the ghost field, under which the effective action is invariant, as

$$
\begin{aligned}
& c \longrightarrow e^{\Lambda} c \\
& \bar{c} \longrightarrow e^{-\Lambda} \bar{c} \\
& \chi \longrightarrow \chi
\end{aligned}
$$

where $\chi=\left\{r, \eta, \theta, \phi, u, \lambda, p_{r}, p_{\eta}, p_{\theta}, p_{\phi}, p_{u}, B\right\}$ and $\Lambda$ is a global scale parameter. The infinitesimal version of the ghost scale transformation can be written as

$$
\begin{aligned}
& s_{g} \chi=0 \\
& s_{g} c=c \\
& s_{g} \bar{c}=-\bar{c} .
\end{aligned}
$$

Noether's conserved charge for above symmetry transformation is calculated as

$$
Q_{g}=i[\dot{\bar{c}} c+\dot{c} \bar{c}]
$$

In addition to above continuous symmetry transformation, the ghost sector respects the following discrete symmetry transformations:

$$
\begin{aligned}
& c \longrightarrow \pm i \bar{c}, \\
& \bar{c} \longrightarrow \pm i c .
\end{aligned}
$$




\section{Geometric Cohomology and Double Hodge Theory}

In this section we study the de Rham cohomological operators [17-19] and their realization in terms of conserved charges which generate the nilpotent symmetries for the theory of a particle on the surface of torus. In particular we point out the similarities between the algebra obeyed by de Rham cohomological operators and that by different BRST conserved charges.

Before we proceed to discuss the analogy, we briefly review the essential features of Hodge theory [50-52]. The de Rham cohomological operators in differential geometry obey the following algebra:

$$
\begin{aligned}
d^{2} & =\delta^{2}=0, \\
\Delta & =(d+\delta)^{2}=d \delta+\delta d \equiv\{d, \delta\} \\
{[\Delta, \delta] } & =0, \\
{[\Delta, d] } & =0,
\end{aligned}
$$

where $d, \delta$, and $\Delta$ are exterior, coexterior, and LaplaceBeltrami operator, respectively. The operators $d$ and $\delta$ are adjoint or dual to each other and $\Delta$ is self-adjoint operator. It is well known that the exterior derivative raises the degree of form by one when it operates on forms (i.e. $d f_{n} \sim f_{n+1}$ ), whereas the dual-exterior derivative lowers the degree of a form by one when it operates on forms (i.e. $\delta f_{n} \sim f_{n-1}$ ). However $\Delta$ does not change the degree of form (i.e. $\Delta f_{n} \sim$ $\left.f_{n}\right) . f_{n}$ denotes an arbitrary $n$-form object.

The Hodge-de Rham decomposition theorem can be stated as follows.

A regular differential form of degree $n(\alpha)$ may be uniquely decomposed into a sum of the harmonic form $(\alpha)_{H}$, exact form $\left(\alpha_{d}\right)$, and coexact form $\left(\alpha_{\delta}\right)$; that is,

$$
\alpha=\alpha_{H}+\alpha_{d}+\alpha_{\delta}
$$

where $\alpha \in H^{n}, \alpha_{s} \in \Lambda_{\delta}^{n}$ and $\alpha_{d} \in \Lambda_{d}^{n}$

The generators of all the nilpotent symmetry transformations satisfy the following algebra [50-52]:

$$
\begin{array}{r}
Q_{b}^{2}=0, \\
Q_{a b}^{2}=0, \\
Q_{d}^{2}=0, \\
Q_{a d}^{2}=0 \\
\left\{Q_{b}, Q_{a b}\right\}=0, \\
\left\{Q_{d}, Q_{a d}\right\}=0, \\
\left\{Q_{b}, Q_{a d}\right\}=0 \\
\left\{Q_{d}, Q_{a b}\right\}=0, \\
{\left[Q_{g}, Q_{b}\right]=Q_{b},}
\end{array}
$$

$$
\begin{aligned}
{\left[Q_{g}, Q_{a d}\right] } & =Q_{a d} \\
{\left[Q_{g}, Q_{d}\right] } & =-Q_{d}, \\
{\left[Q_{g}, Q_{a b}\right] } & =-Q_{a b}, \\
{\left[Q_{w}, Q_{r}\right] } & =0 \\
\left\{Q_{b}, Q_{d}\right\} & =-\left\{Q_{a d}, Q_{a b}\right\}=Q_{w} .
\end{aligned}
$$

Here the relations between the conserved charges $Q_{b}$ and $Q_{a d}$ as well as $Q_{a b}$ and $Q_{a d}$ can be found using equation of motions only. This algebra is similar to the algebra satisfied by de Rham cohomological operators of differential geometry given in (50). Comparing (50) and (55) we obtain following analogies

$$
\begin{aligned}
\left(Q_{b}, Q_{a d}\right) & \longrightarrow d, \\
\left(Q_{d}, Q_{a b}\right) & \longrightarrow \delta, \\
Q_{w} & \longrightarrow \Delta .
\end{aligned}
$$

Let $n$ be the ghost number associated with a given state $|\psi\rangle_{n}$ defined in the total Hilbert space of states; that is,

$$
i Q_{g}|\psi\rangle_{n}=n|\psi\rangle_{n}
$$

Then it is easy to verify the following relations:

$$
\begin{aligned}
Q_{g} Q_{b}|\psi\rangle_{n} & =(n+1) Q_{b}|\psi\rangle_{n} \\
Q_{g} Q_{a d}|\psi\rangle_{n} & =(n+1) Q_{a d}|\psi\rangle_{n} \\
Q_{g} Q_{d}|\psi\rangle_{n} & =(n-1) Q_{b}|\psi\rangle_{n} \\
Q_{g} Q_{a b}|\psi\rangle_{n} & =(n-1) Q_{a d}|\psi\rangle_{n} \\
Q_{g} Q_{w}|\psi\rangle_{n} & =n Q_{w}|\psi\rangle_{n}
\end{aligned}
$$

which imply that the ghost numbers of the states $Q_{b}|\psi\rangle_{n}, Q_{d}|\psi\rangle_{n}$ and $Q_{w}|\psi\rangle_{n}$ are $(n+1),(n-1)$ and $n$, respectively. The states $Q_{a b}|\psi\rangle_{n}$ and $Q_{a d}|\psi\rangle_{n}$ have ghost numbers $(n-1)$ and $(n+1)$, respectively. The properties of sets $\left(Q_{b}, Q_{a d}\right)$ and $\left(Q_{d}, Q_{a b}\right)$ are same as of operators $d$ and $\delta$. It is evident from (55) that the set $Q_{b}, Q_{a d}$ raises the ghost number of a state by one and the set $Q_{d}, Q_{a b}$ lowers the ghost number of the same state by one. Keeping the analogy between charges of different nilpotent symmetries and Hodge-de Rham differential operators, we express any arbitrary state $|\psi\rangle_{n}$ in terms of the sets $\left(Q_{b}, Q_{d}, Q_{w}\right)$ and $\left(Q_{a d}, Q_{a b}, Q_{\bar{w}}\right)$ as

$$
\begin{aligned}
& |\psi\rangle_{n}=|w\rangle_{n}+Q_{b}|\chi\rangle_{(n-1)}+Q_{d}|\phi\rangle_{(n+1)} \\
& |\psi\rangle_{n}=|w\rangle_{n}+Q_{a d}|\chi\rangle_{(n-1)}+Q_{a b}|\phi\rangle_{(n+1)},
\end{aligned}
$$


where the most symmetric state is the harmonic state $|w\rangle_{n}$ that satisfies

$$
\begin{gathered}
Q_{w}|w\rangle_{n}=0 \\
Q_{b}|w\rangle_{n}=0 \\
Q_{d}|w\rangle_{n}=0 \\
Q_{a b}|w\rangle_{n}=0 \\
Q_{a d}|w\rangle_{n}=0
\end{gathered}
$$

analogous to (50). Therefore the BRST charges for a particle on a torus form two separate sets of de Rham cohomological operator, namely, $\left\{Q_{b}, Q_{a b}, Q_{w}\right\}$ and $\left\{Q_{d}, Q_{a d}, Q_{\bar{w}}\right\}$. Thus we call the theory of a particle on torus as double Hodge theory. Fermionic charges $Q_{b}, Q_{a b}, Q_{d}$ and $Q_{a d}$ follow the following physicality criteria:

$$
\begin{aligned}
\left.Q_{b} \mid \text { phys }\right\rangle & =0, \\
\left.Q_{a b} \mid \text { phys }\right\rangle & =0 \\
\left.Q_{d} \mid \text { phys }\right\rangle & =0, \\
\left.Q_{a d} \mid \text { phys }\right\rangle & =0
\end{aligned}
$$

which lead to

$$
\begin{aligned}
\left.p_{\lambda} \mid \text { phys }\right\rangle & =0 \\
\left.\left(P_{r}+P_{\eta}\right) \mid \text { phys }\right\rangle & =0 .
\end{aligned}
$$

This is the operator form of the first class constraint which annihilates the physical state as a consequence of physical criteria, which further is consistent with Dirac's method of quantization of a system with first class constraints.

\section{Finite Field BRST Transformations}

In this section we show that these nilpotent symmetries can be generalized by making the parameter finite and field dependent following the work of Joglekar and Mandal [29]. The BRST transformations can be generated from BRST charge using relation $\delta \phi=[\phi, Q] \delta \Lambda$ where $\delta \Lambda$ is infinitesimal anticommuting BRST parameter under which effective action remains invariant. Joglekar and Mandal generalized the anticommuting BRST parameter $\delta \Lambda$ to be finite field dependent but space-time independent parameter $\Theta[\phi]$. Under this generalization the path integral measure varies nontrivially. The Jacobian for these transformations for certain $\Theta[\phi]$ can be calculated by the following way:

$$
D \phi=J(k) D \phi^{\prime}(k)=J(k+d k) D \phi^{\prime}(k+d k),
$$

where $k$ is a numerical parameter whose value lies between 0 and $1(0<k<1)$. Here all the fields are taken to be $k$ dependent. For a field $\phi(x, k), \phi(x, 0)=\phi(x)$ and $\phi(x, k=$ $1)=\phi^{\prime}(x)$.

The invariance of the $S_{\text {eff }}$ under $\phi(x, 0) \rightarrow \phi(x, k)$ is a BRST transformation given by

$$
\phi(0)=\phi(k)-\delta_{b} \phi(k) \Theta[\phi, k] .
$$

$J(k)$ can be replaced by $e^{i S_{1}[\phi(k) ; k]}$ for a certain functional $S_{1}$ which can be determined in each individual case using following condition

$$
\int D \phi(k)\left[\frac{1}{J(k)} \frac{d J(k)}{d k}-i \frac{d S_{1}}{d k}\right] e^{i\left(S_{1}+S_{\mathrm{eff}}\right)}=0,
$$

where $d S_{1} / d k$ is a total derivative of $S_{1}$ with respect to $k$ in which dependence on $\phi(k)$ is also differentiated and the Jacobian can be expressed as $e^{i S_{1}}$ where $S_{1}$ is local functional of fields which satisfies (60) where change in Jacobian is calculated as

$$
\frac{J(k)}{J(k+d k)}=\sum_{\phi} \pm \frac{\delta \phi(x, k+d k)}{\delta \phi(x, k)}=\frac{1}{J(k)} \frac{d J(k)}{d k} d k
$$

\pm is sign for bosonic and fermionic fields $(\phi)$, respectively.

\section{FFBRST for Free Particle on Surface of Torus}

The effective action for the free particle on surface of torus using BFV formulation is written in (19) and its BRST transformation is given by (23). In BRST transformation given by (23), $\delta \Lambda$ is global, infinitesimal, and anticommuting parameter. FFBRST transformation corresponding to this BRST transformation is written as

$$
\begin{aligned}
s_{b} r & =c \Theta, \\
s_{b} \lambda & =-\dot{c} \Theta, \\
s_{b} \eta & =c \Theta \\
s_{b} p_{r} & =0, \\
s_{b} p_{\eta} & =0, \\
s_{b} c & =0 \\
s_{b} \bar{c} & =(\dot{\lambda}-\eta-r) \Theta,
\end{aligned}
$$

where $\Theta$ is finite field dependent, global, and anticommuting parameter. Under this transformation too, effective action is invariant.

Generating functional for this effective theory can be written as

$$
\begin{aligned}
Z_{\psi}= & \int D \Phi \exp \left[i \int d ^ { 4 } x \left[p_{r} \dot{r}+p_{\eta} \dot{\eta}+p_{\theta} \dot{\theta}+p_{\phi} \dot{\phi}-p_{\lambda} \dot{\lambda}-\frac{p_{r}^{2}}{2 m}\right.\right. \\
& -\frac{p_{\theta}^{2}}{2 m(r-\eta)^{2}}-\frac{p_{\phi}^{2}}{2 m(b+(r-\eta) \sin \theta)^{2}} \\
& +\dot{c} p+\dot{\bar{c}} \bar{p}+\lambda\left(p_{r}-p_{\eta}\right) \\
& \left.\left.+2 c \bar{c}-\bar{p} p+p_{\lambda}\left(r+\eta+\frac{p_{\lambda}}{2}\right)\right]\right],
\end{aligned}
$$

where

$$
D \Phi=d r d p_{r} d \theta d p_{\theta} d \phi d p_{\phi} d \eta d p_{\eta} d \lambda d p_{\lambda} d p d \bar{p} d c d \bar{c}
$$


where $D \Phi$ is the path integral measure integrated over total phase space. The finite BRST transformation given above leaves the effective action invariant but path integral measure in generating functional is not invariant under this transformation. It gives rise to a Jacobian in the extended phase space which can be calculated as

$$
\begin{aligned}
& D \Phi=d r d p_{r} d \theta d p_{\theta} d \phi d p_{\phi} d \eta d p_{\eta} d \lambda d p_{\lambda} d p d \bar{p} d c d \bar{c} \\
& =J(k) d r(k) d p_{r}(k) d \theta(k) d p_{\theta}(k) d \phi(k) d p_{\phi}(k) d \eta(k) d p_{\eta}(k) d \lambda(k) \\
& \cdot d p_{\lambda}(k) d u(k) d p_{u}(k) d p(k) d \bar{p}(k) d c(k) d \bar{c}(k) \\
& =J(k+d k) d r(k+d k) d p_{r}(k+d k) d \theta(k+d k) d p_{\theta}(k+d k) d \phi(k+d k) d p_{\phi}(k+d k) d \eta \\
& \cdot(k+d k) d p_{\eta}(k+d k) d \lambda(k+d k) d p_{\lambda}(k+d k) d p(k+d k) d \bar{p}(k+d k) d c(k+d k) d \bar{c}(k+d k) .
\end{aligned}
$$

Write it in compact form as

$$
=\int d^{4} x \sum_{\psi}\left[\frac{\delta \Psi(x, k+d k)}{\delta \Psi(x, k)}\right],
$$

where $\Psi=\left(r, p_{r}, \theta, p_{\theta}, \phi, p_{\phi}, \eta, p_{\eta}, \lambda, p_{\lambda}, p, \bar{p}, c, \bar{c}\right)$, which can be written as

$$
\begin{aligned}
= & 1+d k \int c \frac{\delta \Theta^{\prime}(x, k+d k)}{\delta r(x, k)}-\dot{c} \frac{\delta \Theta(x, k+d k)}{\delta \lambda(x, k)} \\
& +c \frac{\delta \Theta(x, k+d k)}{\delta \eta(x, k)}+(\dot{\lambda}-\eta-r) \frac{\delta \Theta(x, k+d k)}{\delta \bar{c}(x, k)} \\
= & \frac{J(k)}{J(k+d k)}=1-\frac{1}{J(k)} \frac{d J(k)}{d k} d k .
\end{aligned}
$$

Now we consider an example to illustrate the FFBRST formulation. For that purpose we construct finite BRST parameter $\Theta$ obtained

$$
\Theta^{\prime}=i \gamma \int d^{4} y \bar{c}(y, k) p_{\lambda}(y, k)
$$

through

$$
\Theta=\int \Theta^{\prime}(k) d k
$$

The Jacobian change is calculated

$$
\frac{1}{J(k)} \frac{d J(k)}{d k}=i \gamma \int d^{4} y p_{\lambda}^{2}
$$

We make an ansatz for $S_{1}$ as

$$
S_{1}=i \int d^{4} x \xi_{1}(k) p_{\lambda}^{2}
$$

where $\xi_{1}(k)$ is a $k$ dependent arbitrary parameter. Now,

$$
\frac{d S_{1}}{d k}=i \int d^{4} x \xi_{1}^{\prime}(k) p_{\lambda}^{2}
$$

Using condition in (62), we will get $\xi_{1}(k)=\gamma k$. Now the modified generating functional can be written as

$$
\begin{aligned}
Z= & \int D \chi^{\prime}(k) e^{i\left(S_{1}+S_{\mathrm{eff}}\right)} \\
= & \int D \phi^{\prime} \exp \left[i \int d ^ { 4 } x \left[p_{r} \dot{r}+p_{\eta} \dot{\eta}+p_{\theta} \dot{\theta}+p_{\phi} \dot{\phi}-p_{\lambda} \dot{\lambda}\right.\right. \\
& -\frac{p_{r}^{2}}{2 m}-\frac{p_{\theta}^{2}}{2 m(r-\eta)^{2}}-\frac{p_{\phi}^{2}}{2 m(b+(r-\eta) \sin \theta)^{2}} \\
& +\dot{c} p+\dot{\bar{c}} \bar{p}+\lambda\left(p_{r}+p_{\eta}\right)+2 \bar{c}-\bar{p} p \\
& \left.\left.+p_{\lambda}(r+\eta)+\left(\frac{\lambda^{\prime}}{2}+\gamma k\right) p_{\lambda}{ }^{2}\right]\right] .
\end{aligned}
$$

Here generating functional at $k=0$ is the theory for a free particle on a surface of torus with a gauge parameter $\lambda^{\prime}$ and at $k=1$ the generating functional for same theory with a different gauge parameter $\lambda^{\prime \prime}=\lambda^{\prime}+2 \gamma$. These two effective theories with two different gauge parameters on the surface of a torus are related through the FFBRST transformation with finite parameter given in (70). FFBRST transformation is thus helpful in showing the gauge independence of physical quantities.

\section{Conclusions}

BFVsystem: We have used this technique to study all the symmetries of a free particle on the surface of torus. We have constructed nilpotent BRST, dual BRST, anti-BRST, and anti-dual BRST transformations for this system. Dual BRST transformations are also the symmetry of effective action and leave gauge fixing part of the effective action invariant. Interchanging the role of ghost and anti-ghost fields the anti-BRST and anti-dual BRST symmetry transformations is constructed. We have shown that the nilpotent BRST and anti-dual BRST charges are analogous to the exterior derivative operators as the ghost number of the state $|\psi\rangle_{n}$ on the total Hilbert space is increased by one when these 
charges operate on this state and algebra followed by these operators is the same as the algebra obeyed by the de Rham cohomological operators. Similarly the dual BRST and antiBRST charges are analogous to coexterior derivative. The anticommutators of BRST and dual BRST and anti-BRST and anti-dual BRST charges lead to bosonic symmetry. The corresponding charges are analogous to Laplacian operator. Further, this theory has another nilpotent symmetry called ghost symmetry under which the ghost term of the effective action is invariant. We further have shown that this theory behaves as double Hodge theory as the charges for BRST $\left(Q_{b}\right)$ and dual BRST $\left(Q_{d}\right)$ and the charges for the bosonic symmetry generated out of these two symmetries $\left(Q_{w}\right)$ form the algebra for Hodge theory. On the other hand charges for anti-BRST $\left(Q_{a b}\right)$, anti-dual BRST $\left(Q_{a d}\right)$, and $Q_{\bar{w}}$, charge for bosonic symmetry generalized out of these nilpotent symmetries, also satisfy the Hodge algebra. Thus a particle on the surface of the torus has very rich mathematical structure.

We further constructed the FFBRST transformation for this system. By constructing appropriate field dependent parameter we have explicitly shown that such generalized BRST transformations are capable of connecting different theories on torus. It will be interesting to construct finite version dual BRST transformations and study its consequences in studying system with constraints.

\section{Conflicts of Interest}

There are no conflicts of interest regarding the publication of this paper.

\section{Acknowledgments}

Vipul Kumar Pandey acknowledges University Grant Commission (UGC), India, for its financial assistance under CSIRUGC JRF/SRF Scheme.

\section{References}

[1] C. Becchi, A. Rouet, and R. Stora, "The abelian Higgs Kibble model, unitarity of the S-operator," Physics Letters B, vol. 52, no. 3, pp. 344-346, 1974.

[2] C. Becchi, A. Rouet, and R. Stora, "Renormalization of gauge theories," Annals of Physics, vol. 98, no. 2, pp. 287-321, 1976.

[3] I. V. Tyutin, "Gauge invariance in field theory and statistical physics in operator formalism," Lebedev Report N Fian, vol. 39, 1975.

[4] M. Chaichian and N. F. Nelipa, Introduction to gauge field theories, Texts and Monographs in Physics, Springer-Verlag, Berlin, 1984.

[5] J. Polchinski, Superstring Theory and Beyond String Theory, vol. 2 of Cambridge, UK, Cambridge University Press, 1998.

[6] E. Witten, "On background-independent open-string field theory," Physical Review D: Particles, Fields, Gravitation and Cosmology, vol. 46, no. 12, pp. 5467-5473, 1992.

[7] K. Hotta, "Finite temperature systems of brane-antibrane on a torus," Journal of High Energy Physics, vol. 0309, p. 002, 2003.
[8] J. J. Atick and E. Witten, "The Hagedorn transition and the number of degrees of freedom of string theory," Nuclear Physics B, vol. 310, no. 2, pp. 291-334, 1988.

[9] S. S. Gubser, S. Gukov, I. R. Klebanov, M. Rangamani, and E. Witten, "The Hagedorn transition in noncommutative open string theory," Journal of Mathematical Physics, vol. 42, no. 7, pp. 2749-2764, 2001.

[10] M. Aguado, M. Asorey, and A. Wipf, "Nahm transform and moduli spaces of N-models on the torus," Annals of Physics, vol. 298, no. 1, pp. 2-23, 2002.

[11] K.-L. Chan and M. Cvetic, "Massless BPS-saturated states on the two-torus moduli sub-space of heterotic string," Physics Letters. B. Particle Physics, Nuclear Physics and Cosmology, vol. 375, no. 1-4, pp. 98-102, 1996.

[12] S.-T. Hong, "BRST symmetric in free particle system on toric geometry," Modern Physics Letters A, vol. 20, no. 21, pp. 1577$1588,2005$.

[13] M. Lavelle and D. McMullan, "Nonlocal symmetry for QED," Physical Review Letters, vol. 71, no. 23, pp. 3758-3761, 1993.

[14] Z. Tang and D. Finkelstein, "Relativistically covariant symmetry in QED," Physical Review Letters, vol. 73, no. 23, pp. 3055-3057, 1994.

[15] M. Lavelle and D. McMullan, "Lavelle and McMullan reply 1," Physical Review Letters, vol. 75, no. 22, p. 4151, 1995.

[16] P. Bracken, "The Hodge-de Rham decomposition theorem and an application to a partial differential equation," Acta Mathematica Hungarica, vol. 133, no. 4, pp. 332-341, 2011.

[17] S. I. Goldberg, Curvature and Homology, Dover Publications, Inc., Mineola, NY, USA, 1970.

[18] S. Morita, Geometry of Differential Forms, vol. 209 of Translations of Mathematical Monographs, American Mathematical Society, Providence, RI, 2001.

[19] F. W. Warner, Foundations of differentiable manifolds and Lie groups, vol. 94 of Graduate Texts in Mathematics, SpringerVerlag, New York-Berlin, 1983.

[20] T. Eguchi, P. B. Gilkey, and A. J. Hanson, "Gravitation, gauge theories and differential geometry," Physics Reports, vol. 66, no. 6, pp. 213-393, 1980.

[21] K. Nishijima, "The Casimir operator in the representations of BRS algebra," Progress of Theoretical and Experimental Physics, vol. 80, no. 5, pp. 897-904, 1988.

[22] K. Nishijima, "Observable states in the representations of BRS algebra," Progress of Theoretical and Experimental Physics, vol. 80, no. 5, pp. 905-912, 1988.

[23] W. Kalau and J. W. van Holten, "BRST cohomology and BRST gauge fixing," Nuclear Physics B, vol. 361, no. 1, pp. 233-252, 1991.

[24] J. W. van Holten, "Becchi-Rouet-Stora-Tyutin cohomology of compact gauge algebras," Physical Review Letters, vol. 64, no. 24, pp. 2863-2865, 1990.

[25] J. W. van Holten, "The BRST complex and the cohomology of compact lie algebras," Nuclear Physics B, vol. 339, no. 1, pp. 158176,1990

[26] H. Aratyn, "BRS cohomology in string theory: geometry of abelization and the quartet mechanism," Journal of Mathematical Physics, vol. 31, no. 5, article 1240, 1990.

[27] E. Harikumar, R. P. Malik, and M. Sivakumar, "Hodge decomposition theorem for abelian two-form gauge theory," Journal of Physics A: Mathematical and General, vol. 33, no. 40, pp. 71497163, 2000.

[28] J. Wess and B. Zumino, "Consequences of anomalous ward identities," Physics Letters B, vol. 37, no. 1, pp. 95-97, 1971. 
[29] S. D. Joglekar and B. P. Mandal, "Finite field-dependent BRS transformations," Physical Review D: Particles, Fields, Gravitation and Cosmology, vol. 51, no. 4, pp. 1919-1928, 1995.

[30] S. D. Joglekar and A. Misra, "Relating Green's functions in axial and Lorentz gauges using finite field-dependent BRS transformations," Journal of Mathematical Physics, vol. 41, no. 4, pp. 1755-1767, 2000.

[31] M. Plyushchay, "Hidden nonlinear supersymmetries in pure parabosonic systems," International Journal of Modern Physics A, vol. 15, no. 23, pp. 3679-3698, 2000.

[32] S. D. Joglekar and A. Misra, "A Derivation of the Correct Treatment of $1 /(\eta \cdot k)^{p}$ Singularities in Axial Gauges," Modern Physics Letters A, vol. 14, no. 30, pp. 2083-2092, 1999.

[33] S. D. Joglekar and A. Misra, "Wilson loop and the treatment of axial gauge poles," Modern Physics Letters A, vol. 15, no. 08, pp. 541-546, 2000.

[34] S. D. Joglekar, "Green's functions in axial- and lorentz-type gauges and brs transformations," Modern Physics Letters A, vol. 15, no. 04, pp. 245-252, 2000.

[35] S. D. Joglekar and B. P. Mandal, "Application of finite fielddependent BRS transformations to problems of the Coulomb gauge," International Journal of Modern Physics A, vol. 17, pp. 1279-1300, 2002.

[36] S. D. Joglekar and J. Mod, “Connecting Green's Functions in an Arbitrary Pair of Gauges and an Application to Planar Gauges Satish D. Joglekar," International Journal of Modern Physics A, vol. 16, pp. 5043-5060, 2001.

[37] R. Banerjee and B. P. Mandal, "Quantum gauge symmetry from finite field dependent BRST transformations," Physics Letters. B. Particle Physics, Nuclear Physics and Cosmology, vol. 488, no. 1, pp. 27-30, 2000.

[38] R. S. Bandhu and S. D. Joglekar, "Finite field-dependent BRS transformation and axial gauges," Journal of Physics A: Mathematical and General, vol. 31, no. 18, pp. 4217-4224, 1998.

[39] S. Upadhyay and B. P. Mandal, "Relating Gribov-Zwanziger theory to effective Yang-Mills theory," EPL (Europhysics Letters), vol. 93, no. 3, Article ID 31001, 2011.

[40] S. Deguchi, V. K. Pandey, and B. P. Mandal, "Maximal Abelian gauge and a generalized BRST transformation," Physics Letters $B$, vol. 756, pp. 394-399, 2016.

[41] S. Upadhyay and B. P. Mandal, "Generalized BRST symmetry for arbitrary spin conformal field theory," Physics Letters. B. Particle Physics, Nuclear Physics and Cosmology, vol. 744, pp. 231-236, 2015.

[42] S. Upadhyay, A. Reshetnyak, and B. P. Mandal, "Comments on interactions in the SUSY models," The European Physical Journal C, vol. 76, no. 7, article no. 391, 2016.

[43] M. Faizal, S. Upadhyay, and B. P. Mandal, "Finite fielddependent BRST symmetry for ABJM theory in $\mathrm{N}=1$ superspace," Physics Letters B, vol. 738, pp. 201-205, 2014.

[44] P. A. M. Dirac, Lectures on Quantum Mechanics, Belfer Graduate School of Science, Yeshiva University Press, New York, NY, USA, 1964.

[45] M. Henneaux, "Hamiltonian form of the path integral for theories with a gauge freedom," Physics Reports, vol. 126, no. 1, pp. 1-66, 1985.

[46] M. Henneaux and C. Teitelboim, Quantization of Gauge Systems, Princeton University Press, 1992.

[47] K. Sundermeyer, Constrained Dynamics, vol. 169 of Lecture Notes in Physics, Springer, Berlin, Germany, 1982.
[48] I. A. Batalin and E. S. Fradkin, "A generalized canonical formalism and quantization of reducible gauge theories," Physics Letters B, vol. 122, no. 2, pp. 157-164, 1983.

[49] E. S. Fradkin and G. A. Vilkovisky, "Quantization of relativistic systems with constraints," Physics Letters B, vol. 55, no. 2, pp. 224-226, 1975.

[50] S. Upadhyay and B. P. Mandal, "The model for self-dual chiral bosons as a Hodge theory," The European Physical Journal C, vol. 71, no. 9, article 1759, 2011.

[51] S. Upadhyay and B. P. Mandal, "Noncommutative gauge theories: model for Hodge theory," International Journal of Modern Physics A, vol. 28, no. 25, 1350122, 15 pages, 2013.

[52] S. Upadyay, "Quantum gauge symmetry from finite field dependent BRST transformations," High Energy Physics - Theory, 2013.

[53] S. K. Rai and B. P. Mandal, "Finite nilpotent BRST transformations in Hamiltonian formulation," International Journal of Theoretical Physics, vol. 52, no. 10, pp. 3512-3521, 2013.

[54] R. Kumar, "Off-shell nilpotent (anti-)BRST symmetries for a free particle system on a toric geometry: superfield formalism," EPL (Europhysics Letters), 106, 51001, 2014. 



Submit your manuscripts at

https://www.hindawi.com
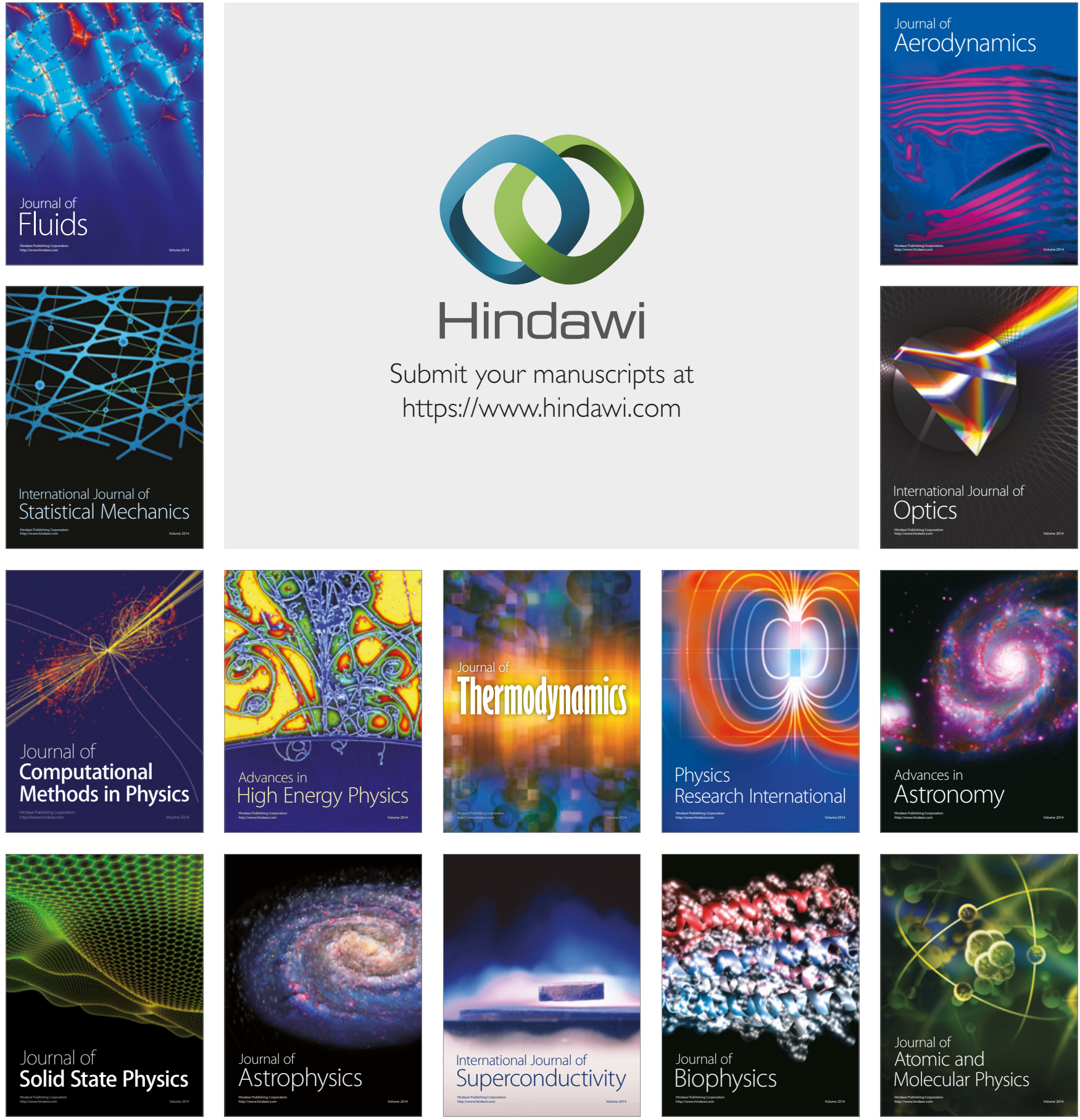